\title{
ON INCREASING OF DENSITY OF ELEMENTS IN A MULTIVIBRATOR ON BIPOLAR TRANSISTORS
}

\author{
E.L. Pankratov ${ }^{1}$ and E.A. Bulaeva ${ }^{1,2}$ \\ ${ }^{1}$ Nizhny Novgorod State University, 23 Gagarin avenue, Nizhny Novgorod, 603950, \\ Russia \\ ${ }^{2}$ Nizhny Novgorod State University of Architecture and Civil Engineering, 65 Il'insky \\ street, Nizhny Novgorod, 603950, Russia
}

\begin{abstract}
In this paper we consider an approach to increase density of elements of a multivibrator on bipolar transistors. The considered approach based on manufacturing a heterostructure with necessity configuration, doping by diffusion or ion implantation of required areas to manufacture the required type of conductivity ( $p$ or $n$ ) in the areas and optimization of annealing of dopant and/or radiation defects to manufacture more compact distributions of concentrations of dopants. We also introduce an analytical approach to prognosis technological process.
\end{abstract}

\section{KEYWORDS}

Bipolar transistors, multivibrator, increasing of density of elements of multivibrator

\section{INTRODUCTION}

In the present time actual questions of solid state electronic devices are increasing of density of elements of integrated circuits (in this situation it is necessary to decrease dimensions of these elements), performance and reliability [1-9]. In this paper we consider an approach to manufacture more compact multivibrator based on bipolar heterotransistors. Framework the approach we consider a heterostructure with two layers, which consist of a substrate and an epitaxial layer (see Fig. 1). The epitaxial layer includes into itself several sections manufactured by using another materials (see Fig. 1). These sections have been doped by diffusion or ion implantation to obtain required type of conductivity ( $n$ or $p$ ). The doping gives a possibility to manufacture bipolar transistor framework the considered heterostructure with account the Fig. 1. After that we consider annealing of dopant and/or radiation defects. The annealing should be optimized to manufacture more compact distributions of concentrations of dopant. Main aim of the present paper is determination of conditions, which correspond to increasing of compactness and at the same time to increasing of homogeneity of distribution of concentration of dopant in enriched area.

\section{Method OF SOlution}

We determine spatio-temporal distribution of concentration of dopant by solving the following boundary problem

$$
\frac{\partial C(x, y, z, t)}{\partial t}=\frac{\partial}{\partial x}\left[D \frac{\partial C(x, y, z, t)}{\partial x}\right]+\frac{\partial}{\partial y}\left[D \frac{\partial C(x, y, z, t)}{\partial y}\right]+\frac{\partial}{\partial z}\left[D \frac{\partial C(x, y, z, t)}{\partial z}\right]
$$

with boundary and initial conditions

DOI : $10.5121 /$ ijcsitce.2016.3302 
International Journal of Computational Science, Information Technology and Control Engineering (IJCSITCE) Vol.3, No.3, July 2016

$$
\begin{gathered}
\left.\frac{\partial C(x, y, z, t)}{\partial x}\right|_{x=0}=0,\left.\frac{\partial C(x, y, z, t)}{\partial x}\right|_{x=L_{x}}=0,\left.\frac{\partial C(x, y, z, t)}{\partial y}\right|_{y=0}=0,\left.\frac{\partial C(x, y, z, t)}{\partial y}\right|_{x=L_{y}}=0, \\
\left.\left.\frac{\partial C(x, y, z, t)}{\partial z}\right|_{z=0}=0,\left.\frac{\partial C(x, y, z, t)}{\partial z}\right|_{x=L_{z}}=0, C x, y, z, 0\right)=f_{C}(x, y, z) .
\end{gathered}
$$

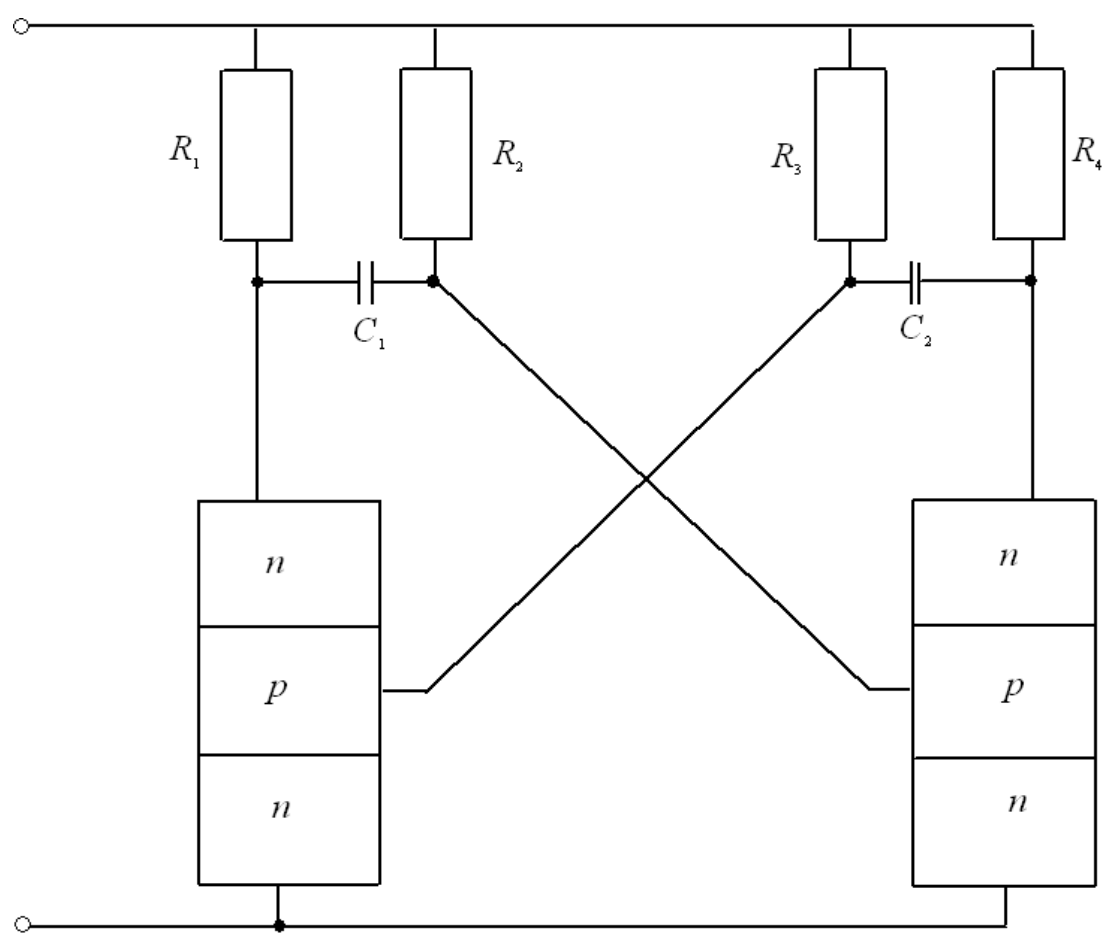

Fig. 1a. Structure of a transistor multivibrator. View from top

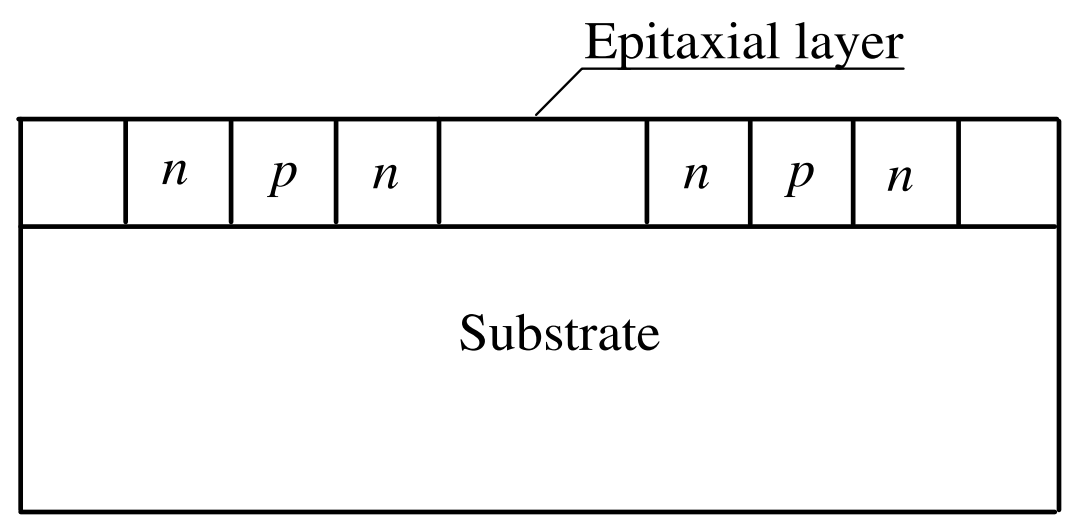

Fig. $1 b$. Heterostructure with two layers and sections in the epitaxial layer, which has been used for manufacture a transistor multivibrator. View from side

Here $C(x, y, z, t)$ is the spatio-temporal distribution of concentration of dopant; $T$ is the temperature of annealing; $D_{C}$ is the dopant diffusion coefficient. Value of dopant diffusion coefficient depends on properties of materials of heterostructure, speed of heating and cooling of heterostructure (with account Arrhenius law). Dependences of dopant diffusion coefficient on parameters could be approximated by the following relation [10-12] 


$$
D_{C}=D_{L}(x, y, z, T)\left[1+\xi \frac{C^{\gamma}(x, y, z, t)}{P^{\gamma}(x, y, z, T)}\right]\left[1+\varsigma_{1} \frac{V(x, y, z, t)}{V^{*}}+\varsigma_{2} \frac{V^{2}(x, y, z, t)}{\left(V^{*}\right)^{2}}\right],
$$

where $D_{L}(x, y, z, T)$ is the spatial (due to presents several layers in heterostructure) and temperature (due to Arrhenius law) dependences of dopant diffusion coefficient; $P(x, y, z, T)$ is the limit of solubility of dopant; parameter $\gamma$ depends on properties of materials and could be integer in the following interval $\gamma \in[1,3][10] ; V(x, y, z, t)$ is the spatio-temporal distribution of concentration of radiation vacancies; $V^{*}$ is the equilibrium distribution of concentration of vacancies.

Concentrational dependence of dopant diffusion coefficient has been described in details in [10]. Using diffusion type of doping did not generation radiation defects. In this situation $\zeta_{1}=\zeta_{2}=0$. We determine the spatio-temporal distributions of concentrations of radiation defects by solving the following system of equations $[11,12]$

$$
\begin{aligned}
& \frac{\partial I(x, y, z, t)}{\partial t}=\frac{\partial}{\partial x}\left[D_{I}(x, y, z, T) \frac{\partial I(x, y, z, t)}{\partial x}\right]+\frac{\partial}{\partial y}\left[D_{I}(x, y, z, T) \frac{\partial I(x, y, z, t)}{\partial y}\right]-k_{I, I}(x, y, z, T) \times \\
& \quad \times I^{2}(x, y, z, t)+\frac{\partial}{\partial z}\left[D_{I}(x, y, z, T) \frac{\partial I(x, y, z, t)}{\partial z}\right]-k_{I, V}(x, y, z, T) I(x, y, z, t) V(x, y, z, t) \quad(4) \\
& \frac{\partial V(x, y, z, t)}{\partial t}=\frac{\partial}{\partial x}\left[D_{V}(x, y, z, T) \frac{\partial V(x, y, z, t)}{\partial x}\right]+\frac{\partial}{\partial y}\left[D_{V}(x, y, z, T) \frac{\partial V(x, y, z, t)}{\partial y}\right]-k_{V, V}(x, y, z, T) \times \\
& \quad \times V^{2}(x, y, z, t)+\frac{\partial}{\partial z}\left[D_{V}(x, y, z, T) \frac{\partial V(x, y, z, t)}{\partial z}\right]-k_{I, V}(x, y, z, T) I(x, y, z, t) V(x, y, z, t)
\end{aligned}
$$

with boundary and initial conditions

$$
\begin{gathered}
\left.\frac{\partial \rho(x, y, z, t)}{\partial x}\right|_{x=0}=0,\left.\frac{\partial \rho(x, y, z, t)}{\partial x}\right|_{x=L_{x}}=0,\left.\frac{\partial \rho(x, y, z, t)}{\partial y}\right|_{y=0}=0,\left.\frac{\partial \rho(x, y, z, t)}{\partial y}\right|_{y=L_{y}}=0, \\
\left.\frac{\partial \rho(x, y, z, t)}{\partial z}\right|_{z=0}=0,\left.\frac{\partial \rho(x, y, z, t)}{\partial z}\right|_{z=L_{z}}=0, \rho(x, y, z, 0)=f_{\rho}(x, y, z) .
\end{gathered}
$$

Here $\rho=I, V ; I(x, y, z, t)$ is the spatio-temporal distribution of concentration of radiation interstitials; $D_{\rho}(x, y, z, T)$ are the diffusion coefficients of point radiation defects; terms $V^{2}(x, y, z, t)$ and $I^{2}(x, y, z, t)$ correspond to generation divacancies and diinterstitials; $k_{I, V}(x, y, z, T)$ is the parameter of recombination of point radiation defects; $k_{I, I}(x, y, z, T)$ and $k_{V, V}(x, y, z, T)$ are the parameters of generation of simplest complexes of point radiation defects.

We determine the spatio-temporal distributions of concentrations of divacancies $\Phi_{V}(x, y, z, t)$ and dinterstitials $\Phi_{I}(x, y, z, t)$ by solving the following system of equations [11,12]

$$
\begin{aligned}
& \frac{\partial \Phi_{I}(x, y, z, t)}{\partial t}=\frac{\partial}{\partial x}\left[D_{\Phi I}(x, y, z, T) \frac{\partial \Phi_{I}(x, y, z, t)}{\partial x}\right]+\frac{\partial}{\partial y}\left[D_{\Phi I}(x, y, z, T) \frac{\partial \Phi_{I}(x, y, z, t)}{\partial y}\right]+ \\
& +\frac{\partial}{\partial z}\left[D_{\Phi I}(x, y, z, T) \frac{\partial \Phi_{I}(x, y, z, t)}{\partial z}\right]+k_{I, I}(x, y, z, T) I^{2}(x, y, z, t)-k_{I}(x, y, z, T) I(x, y, z, t)
\end{aligned}
$$


International Journal of Computational Science, Information Technology and Control Engineering (IJCSITCE) Vol.3, No.3, July 2016

$$
\begin{aligned}
& \frac{\partial \Phi_{V}(x, y, z, t)}{\partial t}=\frac{\partial}{\partial x}\left[D_{\Phi V}(x, y, z, T) \frac{\partial \Phi_{V}(x, y, z, t)}{\partial x}\right]+\frac{\partial}{\partial y}\left[D_{\Phi V}(x, y, z, T) \frac{\partial \Phi_{V}(x, y, z, t)}{\partial y}\right]+ \\
& +\frac{\partial}{\partial z}\left[D_{\Phi V}(x, y, z, T) \frac{\partial \Phi_{V}(x, y, z, t)}{\partial z}\right]+k_{V, V}(x, y, z, T) V^{2}(x, y, z, t)-k_{V}(x, y, z, T) V(x, y, z, t)
\end{aligned}
$$

with boundary and initial conditions

$$
\begin{gathered}
\left.\frac{\partial \Phi_{\rho}(x, y, z, t)}{\partial x}\right|_{x=0}=0,\left.\frac{\partial \Phi_{\rho}(x, y, z, t)}{\partial x}\right|_{x=L_{x}}=0,\left.\frac{\partial \Phi_{\rho}(x, y, z, t)}{\partial y}\right|_{y=0}=0,\left.\frac{\partial \Phi_{\rho}(x, y, z, t)}{\partial y}\right|_{y=L_{y}}=0, \\
\left.\frac{\partial \Phi_{\rho}(x, y, z, t)}{\partial z}\right|_{z=0}=0,\left.\frac{\partial \Phi_{\rho}(x, y, z, t)}{\partial z}\right|_{z=L_{z}}=0, \Phi_{I}(x, y, z, 0)=f_{\Phi I}(x, y, z), \Phi_{V}(x, y, z, 0)=f_{\Phi V}(x, y, z) .(7)
\end{gathered}
$$

Here $D_{\Phi \rho}(x, y, z, T)$ are the diffusion coefficients of the above complexes of radiation defects; $k_{I}(x, y, z, T)$ and $k_{V}(x, y, z, T)$ are the parameters of decay of these complexes.

To determine spatio-temporal distribution of concentration of dopant we transform the Eq.(1) to the following integro-differential form

$$
\begin{gathered}
\frac{x y z}{L_{x} L_{y} L_{z}} \int_{L_{x} L_{y} L_{z}}^{x} \int_{z}^{z} C(u, v, w, t) d w d v d u=\int_{0}^{t} \int_{L_{y} L_{z}}^{y} \int_{L} D_{L}(x, v, w, T)\left[1+\varsigma_{1} \frac{V(x, v, w, \tau)}{V^{*}}+\varsigma_{2} \frac{V^{2}(x, v, w, \tau)}{\left(V^{*}\right)^{2}}\right] \times \\
\times\left[1+\xi \frac{C^{\gamma}(x, v, w, \tau)}{P^{\gamma}(x, v, w, T)}\right] \frac{\partial C(x, v, w, \tau)}{\partial x} d \tau \frac{y z}{L_{y} L_{z}}+\frac{x z}{L_{x} L_{z}} \int_{0}^{t} \int_{L_{x} L_{z}}^{x} D_{L} D_{L}(u, y, w, T) \frac{\partial C(u, y, w, \tau)}{\partial y} \times \\
\times\left[1+\varsigma_{1} \frac{V(u, y, w, \tau)}{V^{*}}+\varsigma_{2} \frac{V^{2}(u, y, w, \tau)}{\left(V^{*}\right)^{2}}\right]\left[1+\xi \frac{C^{\gamma}(u, y, w, \tau)}{P^{\gamma}(x, y, z, T)}\right] d \tau+\frac{x y}{L_{x} L_{y}} \int_{0}^{t} \int_{L_{x} L_{y}}^{x} \int_{L_{y}}^{y} D_{L}(u, v, z, T) \times \\
\times\left[1+\varsigma_{1} \frac{V(u, v, z, \tau)}{V^{*}}+\varsigma_{2} \frac{V^{2}(u, v, z, \tau)}{\left(V^{*}\right)^{2}}\right]\left[1+\xi \frac{C^{\gamma}(u, v, z, \tau)}{P^{\gamma}(x, y, z, T)}\right] \frac{\partial C(u, v, z, \tau)}{\partial z} d \tau+\frac{x y z}{L_{x} L_{y} L_{z}} \times \\
\times \int_{L_{x} L_{y} L_{z}}^{x} \int_{z}^{z} f(u, v, w) d w d v d u .
\end{gathered}
$$

We determine solution of the above equation by using Bubnov-Galerkin approach [13]. Framework the approach we determine solution of the Eq.(1a) as the following series

$$
C_{0}(x, y, z, t)=\sum_{n=0}^{N} a_{n C} c_{n}(x) c_{n}(y) c_{n}(z) e_{n C}(t),
$$

where $e_{n C}(t)=\exp \left[-\pi^{2} n^{2} D_{0 c} t\left(L_{x}^{-2}+L_{y}^{-2}+L_{z}^{-2}\right)\right], c_{n}(\chi)=\cos \left(\pi n \chi / L_{\chi}\right)$. The above series includes into itself finite number of terms $N$. The considered series is similar with solution of linear Eq.(1) (i.e. with $\xi=0$ ) and averaged dopant diffusion coefficient $D_{0}$. Substitution of the series into Eq.(1a) leads to the following result

$$
\begin{aligned}
& \frac{x y z}{\pi^{2}} \sum_{n=1}^{N} \frac{a_{C}}{n^{3}} s_{n}(x) s_{n}(y) s_{n}(z) e_{n C}(t)=-\int_{0}^{t} \int_{L_{y} L_{z}}^{y} z\left\{1+\left[\sum_{n=1}^{N} a_{n C} c_{n}(x) c_{n}(v) c_{n}(w) e_{n C}(\tau)\right]^{\gamma} \frac{\xi}{P^{\gamma}(x, v, w, T)}\right\} \times \\
& \times\left[1+\varsigma_{1} \frac{V(x, v, w, \tau)}{V^{*}}+\varsigma_{2} \frac{V^{2}(x, v, w, \tau)}{\left(V^{*}\right)^{2}}\right] D_{L}(x, v, w, T) \sum_{n=1}^{N} n a_{n C} s_{n}(x) c_{n}(v) c_{n}(w) e_{n C}(\tau) d \tau \frac{y z}{L_{y} L_{z}}-
\end{aligned}
$$


International Journal of Computational Science, Information Technology and Control Engineering (IJCSITCE) Vol.3, No.3, July 2016

$$
\begin{aligned}
& -\frac{x z}{L_{x} L_{z}} \int_{0}^{t} \int_{L_{x} L_{z}}^{x} \int_{z}^{z}\left[1+\varsigma_{1} \frac{V(u, y, w, \tau)}{V^{*}}+\varsigma_{2} \frac{V^{2}(u, y, w, \tau)}{\left(V^{*}\right)^{2}}\right]\left\{1+\left[\sum_{m=1}^{N} a_{m C} c_{m}(u) c_{m}(y) c_{m}(w) e_{m C}(\tau)\right]^{\gamma} \times\right. \\
& \left.\times \frac{\xi}{P^{\gamma}(u, y, w, T)}\right\} D_{L}(u, y, w, T) \sum_{n=1}^{N} n a_{n C} c_{n}(u) s_{n}(y) c_{n}(w) e_{n C}(\tau) d \tau-\frac{x y}{L_{x} L_{y}} \int_{0}^{t} \int_{L_{x} L_{y}}^{x} \int_{y}^{y} D_{L}(u, v, z, T) \times \\
& \times\left\{1+\frac{\xi}{P^{\gamma}(u, v, z, T)}\left[\sum_{n=1}^{N} a_{n C} c_{n}(u) c_{n}(v) c_{n}(z) e_{n C}(\tau)\right]^{\gamma}\right\}\left[1+\varsigma_{1} \frac{V(u, v, z, \tau)}{V^{*}}+\varsigma_{2} \frac{V^{2}(u, v, z, \tau)}{\left(V^{*}\right)^{2}}\right] \times \\
& \times \sum_{n=1}^{N} n a_{n C} c_{n}(u) c_{n}(v) s_{n}(z) e_{n C}(\tau) d \tau+\frac{x y z}{L_{x} L_{y} L_{z}} \int_{L_{x} L_{y} L_{z}}^{x} \int_{z}^{z} f(u, v, w) d w d v d u,
\end{aligned}
$$

where $s_{n}(\chi)=\sin \left(\pi n \chi / L_{\chi}\right)$. We determine coefficients $a_{n}$ by using orthogonality condition of terms of the considered series framework scale of heterostructure. The condition gives us possibility to obtain relations for calculation of parameters $a_{n}$ for any quantity of terms $N$. In the common case the relations could be written as

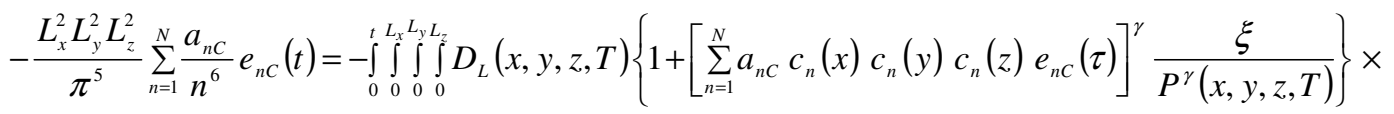

$$
\begin{aligned}
& \times \frac{L_{y} L_{z}}{2 \pi^{2}}\left[1+\varsigma_{1} \frac{V(x, y, z, \tau)}{V^{*}}+\varsigma_{2} \frac{V^{2}(x, y, z, \tau)}{\left(V^{*}\right)^{2}}\right] \sum_{n=1}^{N} \frac{a_{n C}}{n} s_{n}(2 x) c_{n}(y)\left\{y s_{n}(y)+\frac{L_{y}}{\pi n}\left[c_{n}(y)-1\right]\right\} \times \\
& \times c_{n}(z) e_{n C}(\tau)\left\{z s_{n}(z)+\frac{L_{z}}{\pi n}\left[c_{n}(z)-1\right]\right\} d z d y d x d \tau-\int_{0}^{t} \int_{0}^{L_{L}} \int_{0}^{L_{y}} \int_{0}^{L_{z}}\left\{\left[\sum_{n=1}^{N} a_{n C} c_{n}(x) c_{n}(y) c_{n}(z) e_{n C}(\tau)\right]^{\gamma} \times\right. \\
& \left.\times \frac{\xi}{P^{\gamma}(x, y, z, T)}+1\right\} D_{L}(x, y, z, T)\left[1+\varsigma_{1} \frac{V(x, y, z, \tau)}{V^{*}}+\varsigma_{2} \frac{V^{2}(x, y, z, \tau)}{\left(V^{*}\right)^{2}}\right]\left[1+\varsigma_{1} \frac{V(x, y, z, \tau)}{V^{*}}+\right. \\
& \left.+\varsigma_{2} \frac{V^{2}(x, y, z, \tau)}{\left(V^{*}\right)^{2}}\right] \sum_{n=1}^{N} \frac{a_{n C} L_{z}}{2 \pi^{2} n}\left\{x s_{n}(x)+\frac{L_{x}}{\pi n}\left[c_{n}(x)-1\right]\right\}\left\{z s_{n}(z)+\frac{L_{z}}{\pi n}\left[c_{n}(z)-1\right]\right\} c_{n}(x) s_{n}(2 y) c_{n}(z) \times \\
& \times e_{n C}(\tau) d z d y d x d \tau-\frac{L_{x} L_{y}}{2 \pi^{2}} \times \int_{0}^{t} \int_{0}^{L_{x}} \int_{0}^{L_{y} L_{z}} \int_{0}\left\{1+\left[\sum_{n=1}^{N} a_{n C} c_{n}(x) c_{n}(y) c_{n}(z) e_{n C}(\tau)\right]^{\gamma} \frac{\xi}{P^{\gamma}(x, y, z, T)}\right\} \times \\
& \times D_{L}(x, y, z, T)\left[1+\varsigma_{2} \frac{V^{2}(x, y, z, \tau)}{\left(V^{*}\right)^{2}}+\varsigma_{1} \frac{V(x, y, z, \tau)}{V^{*}} \sum_{n=1}^{N} \frac{a_{n C}}{n} c_{n}(x)\left\{x s_{n}(x)+\frac{L_{x}}{\pi n}\left[c_{n}(x)-1\right]\right\} \times\right. \\
& \times c_{n}(y) s_{n}(z)\left\{y s_{n}(y)+\frac{L_{y}}{\pi n}\left[c_{n}(y)-1\right]\right\} e_{n C}(\tau) d z d y d x d \tau+\sum_{n=1}^{N} \int_{0}^{L_{x}}\left\{x s_{n}(x)+\frac{L_{x}}{\pi n}\left[c_{n}(x)-1\right]\right\} \times \\
& \times \int_{0}^{L_{y}}\left\{y s_{n}(y)+\frac{L_{y}}{\pi n}\left[c_{n}(y)-1\right]\right\} \int_{0}^{L_{z}}\left\{z s_{n}(z)+\frac{L_{z}}{\pi n}\left[c_{n}(z)-1\right]\right\} f(x, y, z) d z d y d x .
\end{aligned}
$$

As an example for $\gamma=0$ we obtain

$$
\begin{aligned}
& a_{n C}=\int_{0}^{L_{x}} \int_{0}^{L_{y}}\left\{y s_{n}(y)+\frac{L_{y}}{\pi n}\left[c_{n}(y)-1\right]\right\} \int_{0}^{L_{z}}\left\{z s_{n}(z)+\frac{L_{y}}{\pi n}\left[c_{n}(z)-1\right]\right\} f(x, y, z) d z d y\left\{x s_{n}(x)+\frac{L_{x}}{\pi n} \times\right. \\
& \left.\times\left[c_{n}(x)-1\right]\right\} d x\left(\frac { n } { 2 } \left\{\int _ { 0 } ^ { t } \int _ { 0 } ^ { L _ { x } } s _ { n } ( 2 x ) \int _ { 0 } ^ { L _ { y } } c _ { n } ( y ) \int _ { 0 } ^ { L _ { z } } c _ { n } ( z ) D _ { L } ( x , y , z , T ) \{ z s _ { n } ( z ) + \frac { L _ { y } } { \pi n } [ c _ { n } ( z ) - 1 ] \} \left\{y s_{n}(y)+\right.\right.\right. \\
& \left.+\frac{L_{y}}{\pi n}\left[c_{n}(y)-1\right]\right\}\left[1+\varsigma_{1} \frac{V(x, y, z, \tau)}{V^{*}}+\varsigma_{2} \frac{V^{2}(x, y, z, \tau)}{\left(V^{*}\right)^{2}}\right]\left[1+\frac{\xi}{P^{\gamma}(x, y, z, T)}\right] c_{n}(z) d z d y d x \times
\end{aligned}
$$


International Journal of Computational Science, Information Technology and Control Engineering (IJCSITCE) Vol.3, No.3, July 2016

$$
\begin{aligned}
& \times e_{n C}(\tau) d \tau+\int_{0}^{t} e_{n C}(\tau) \int_{0}^{L_{x}} c_{n}(x)\left\{x s_{n}(x)+\frac{L_{y}}{\pi n}\left[c_{n}(x)-1\right]\right\} \int_{0}^{L_{y}} s_{n}(2 y) \int_{0}^{L_{z}} c_{n}(z)\left\{z s_{n}(z)+\frac{L_{y}}{\pi n}\left[c_{n}(z)-1\right]\right\} \times \\
& \times D_{L}(x, y, z, T)\left[1+\frac{\xi}{P^{\gamma}(x, y, z, T)}\right]\left[1+\varsigma_{1} \frac{V(x, y, z, \tau)}{V^{*}}+\varsigma_{2} \frac{V^{2}(x, y, z, \tau)}{\left(V^{*}\right)^{2}}\right] d z d y d x d \tau+ \\
& +\int_{0}^{t} e_{n C}(\tau) \int_{0}^{L_{x}} c_{n}(x)\left\{x s_{n}(x)+\frac{L_{x}}{\pi n}\left[c_{n}(x)-1\right]\right\} \int_{0}^{L_{y}} c_{n}(y)\left\{y s_{n}(y)+\frac{L_{y}}{\pi n}\left[c_{n}(y)-1\right]\right\} \int_{0}^{L_{z}} D_{L}(x, y, z, T) \times \\
& \left.\left.\times s_{n}(2 z)\left[1+\frac{\xi}{P^{\gamma}(x, y, z, T)}\right]\left[1+\varsigma_{2} \frac{V^{2}(x, y, z, \tau)}{\left(V^{*}\right)^{2}}+\varsigma_{1} \frac{V(x, y, z, \tau)}{V^{*}}\right] d z d y d x d \tau\right\}-\frac{L_{z}^{2} L_{z}^{2} L_{z}^{2}}{\pi^{5} n^{6}}\right)^{-1} .
\end{aligned}
$$

For $\gamma=1$ one can obtain the following relation to determine required parameters

$$
a_{n C}=-\frac{\beta_{n}}{2 \alpha_{n}} \pm \sqrt{\beta_{n}^{2}+4 \alpha_{n} \int_{0}^{L_{x}} c_{n}(x) \int_{0}^{L_{y}} c_{n}(y) \int_{0}^{L_{z}} c_{n}(z) f(x, y, z) d z d y d x}
$$

where $\alpha_{n}=\frac{\xi L_{y} L_{z}}{2 \pi^{2} n} \int_{0}^{t} \int_{0}^{L_{x}} s_{n}(2 x) \int_{0}^{L_{y}} c_{n}(y) \int_{0}^{L_{z}} \frac{D_{L}(x, y, z, T)}{P(x, y, z, T)}\left[1+\varsigma_{1} \frac{V(x, y, z, \tau)}{V^{*}}+\varsigma_{2} \frac{V^{2}(x, y, z, \tau)}{\left(V^{*}\right)^{2}}\right] \times$ $\times c_{n}(z)\left\{y s_{n}(y)+\frac{L_{y}}{\pi n}\left[c_{n}(y)-1\right]\right\}\left\{z s_{n}(z)+\frac{L_{z}}{\pi n}\left[c_{n}(z)-1\right]\right\} d z d y d x e_{n C}(\tau) d \tau+\frac{\xi L_{x} L_{z}}{2 \pi^{2} n} \times$ $\times \int_{0}^{t} e_{n C}(\tau) \int_{0}^{L_{x}} c_{n}(x)\left\{x s_{n}(x)+\frac{L_{x}}{\pi n}\left[c_{n}(x)-1\right]\right\} \int_{0}^{L_{y}} s_{n}(2 y) \int_{0}^{L_{z}} \frac{D_{L}(x, y, z, T)}{P(x, y, z, T)}\left\{z s_{n}(z)-\frac{L_{z}}{\pi n}\left[c_{n}(z)-1\right]\right\} \times$ $\times c_{n}(z)\left[1+\varsigma_{1} \frac{V(x, y, z, \tau)}{V^{*}}+\varsigma_{2} \frac{V^{2}(x, y, z, \tau)}{\left(V^{*}\right)^{2}}\right] d z d y d x d \tau+\frac{\xi L_{x} L_{y}}{2 \pi^{2} n} \int_{0}^{t} e_{n C}(\tau) \int_{0}^{L_{x}} c_{n}(x) \int_{0}^{L_{y}} c_{n}(y) \times$ $\times \int_{0}^{L_{z}} s_{n}(2 z) \frac{D_{L}(x, y, z, T)}{P(x, y, z, T)}\left[1+\varsigma_{1} \frac{V(x, y, z, \tau)}{V^{*}}+\varsigma_{2} \frac{V^{2}(x, y, z, \tau)}{\left(V^{*}\right)^{2}}\right]\left\{x s_{n}(x)+\frac{L_{x}}{\pi n}\left[c_{n}(x)-1\right]\right\}\left\{s_{n}(y) \times\right.$ $\left.\times y+\frac{L_{y}}{\pi n}\left[c_{n}(y)-1\right]\right\} d z d y d x d \tau, \beta_{n}=\frac{L_{y} L_{z}}{2 n \pi^{2}} \int_{0}^{t} e_{n C}(\tau) \int_{0}^{L_{x}} s_{n}(2 x) \int_{0}^{L_{y}} c_{n}(y) \int_{0}^{L_{z}} c_{n}(z) D_{L}(x, y, z, T) \times$ $\times\left[1+\varsigma_{1} \frac{V(x, y, z, \tau)}{V^{*}}+\varsigma_{2} \frac{V^{2}(x, y, z, \tau)}{\left(V^{*}\right)^{2}}\right]\left\{z s_{n}(z)+\frac{L_{z}}{\pi n}\left[c_{n}(z)-1\right]\right\} d z\left\{\frac{L_{y}}{\pi n}\left[c_{n}(y)-1\right]+y s_{n}(y)\right\} \times$ $\times d y d x d \tau+\frac{L_{x} L_{z}}{2 n \pi^{2}} \int_{0}^{t} e_{n C}(\tau) \int_{0}^{L_{x}} c_{n}(x)\left\{x s_{n}(x)+\frac{L_{x}}{\pi n}\left[c_{n}(x)-1\right]\right\} \int_{0}^{L_{y}} s_{n}(2 y) \int_{0}^{L_{z}} c_{n}(z)\left[1+\varsigma_{1} \frac{V(x, y, z, \tau)}{V^{*}}+\right.$ $\left.+\varsigma_{2} \frac{V^{2}(x, y, z, \tau)}{\left(V^{*}\right)^{2}}\right] D_{L}(x, y, z, T)\left\{z s_{n}(z)+\frac{L_{z}}{\pi n}\left[c_{n}(z)-1\right]\right\} d z d y d x d \tau+\frac{L_{x} L_{y}}{2 n \pi^{2}} \int_{0}^{t} e_{n C}(\tau) \times$ $\times \int_{0}^{L_{x}}\left\{x s_{n}(x)+\frac{L_{x}}{\pi n}\left[c_{n}(x)-1\right]\right\} \int_{0}^{L_{y}}\left\{y s_{n}(y)+\frac{L_{y}}{\pi n}\left[c_{n}(y)-1\right]\right\} \int_{0}^{L_{\tilde{z}}}\left[1+\varsigma_{1} \frac{V(x, y, z, \tau)}{V^{*}}+\varsigma_{2} \frac{V^{2}(x, y, z, \tau)}{\left(V^{*}\right)^{2}}\right] \times$ $\times s_{n}(2 z) D_{L}(x, y, z, T) d z c_{n}(y) d y c_{n}(x) d x d \tau-L_{x}^{2} L_{y}^{2} L_{z}^{2} e_{n C}(t) / \pi^{5} n^{6}$.

Analogous way could be used to calculate values of parameters $a_{n}$ for larger values of parameter $\gamma$. However the relations will not be present in the paper because the relations are bulky. Advan- 
tage of the approach is absent of necessity to join dopant concentration on interfaces of heterostructure.

Equations of the system (4) have been also solved by using Bubnov-Galerkin approach. Previously we transform the differential equations to the following integro- differential form

$$
\begin{aligned}
& \frac{x y z}{L_{x} L_{y} L_{z}} \int_{L_{x}}^{x} \int_{L_{y} L_{z}}^{y} \int_{z}^{z} I(u, v, w, t) d w d v d u=\frac{y z}{L_{y} L_{z}} \int_{0}^{t} \int_{L_{y} L_{z}}^{y} \int_{I}^{z} D_{I}(x, v, w, T) \frac{\partial I(x, v, w, \tau)}{\partial x} d w d v d \tau+\frac{x z}{L_{x} L_{z}} \times \\
& \times \int_{0}^{t} \int_{L_{x} L_{z}}^{x} \int_{I}^{z} D_{I}(u, y, w, T) \frac{\partial I(u, y, w, \tau)}{\partial x} d w d u d \tau+\frac{x y}{L_{x} L_{y}} \int_{0}^{t} \int_{L_{x}}^{x} \int_{L_{y}}^{y} D_{I}(u, v, z, T) \frac{\partial I(u, v, z, \tau)}{\partial z} d v d u d \tau-
\end{aligned}
$$

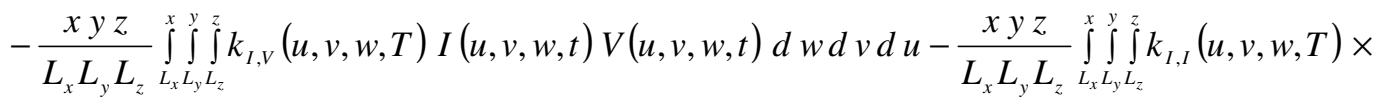

$$
\begin{aligned}
& \times I^{2}(u, v, w, t) d w d v d u+\frac{x y z}{L_{x} L_{y} L_{z}} \int_{L_{x}}^{x} \int_{y}^{y} \int_{L_{z}}^{z} f_{I}(u, v, w) d w d v d u \\
& \frac{x y z}{L_{x} L_{y} L_{z}} \int_{L_{x} L_{y} L_{z}}^{x} \int^{z} V(u, v, w, t) d w d v d u=\frac{y z}{L_{y} L_{z}} \int_{0}^{t} \int_{L_{y} L_{z}}^{y} \int_{V}^{z} D_{V}(x, v, w, T) \frac{\partial V(x, v, w, \tau)}{\partial x} d w d v d \tau+\frac{x z}{L_{x} L_{z}} \times \\
& \times \int_{0}^{t} \int_{L_{x}}^{x} \int_{L_{z}}^{z} D_{V}(u, y, w, T) \frac{\partial V(u, y, w, \tau)}{\partial x} d w d u d \tau+\frac{x y}{L_{x} L_{y}} \int_{0}^{t} \int_{L_{x}}^{x} \int_{L_{y}}^{y} D_{V}(u, v, z, T) \frac{\partial V(u, v, z, \tau)}{\partial z} d v d u d \tau-
\end{aligned}
$$

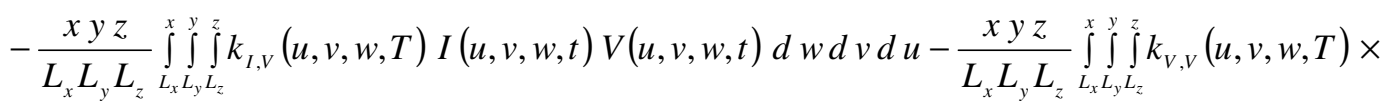

$$
\begin{aligned}
& \times V^{2}(u, v, w, t) d w d v d u+\frac{x y z}{L_{x} L_{y} L_{z}} \int_{L_{x}}^{x} \int_{L_{y} L_{z}}^{y} \int_{V}^{z} f_{V}(u, v, w) d w d v d u .
\end{aligned}
$$

Farther we determine solutions of the above equations as the following series

$$
\rho_{0}(x, y, z, t)=\sum_{n=1}^{N} a_{n \rho} c_{n}(x) c_{n}(y) c_{n}(z) e_{n \rho}(t)
$$

where $a_{n \rho}$ are not yet known coefficients. Substitution of the series into Eqs.(4a) leads to the following results

$$
\begin{aligned}
& \frac{x y z}{\pi^{3}} \sum_{n=1}^{N} \frac{a_{n I}}{n^{3}} s_{n}(x) s_{n}(y) s_{n}(z) e_{n I}(t)=-\frac{y z \pi}{L_{x} L_{y} L_{z}} \sum_{n=1}^{N} a_{n I} \int_{0}^{t} e_{n I}(\tau) \int_{L_{y}}^{y} c_{n}(y) \int_{L_{z}}^{z} c_{n}(z) D_{I}(x, v, w, T) d w d v d \tau \times \\
& \times s_{n}(x)-\frac{x z \pi}{L_{x} L_{y} L_{z}} \sum_{n=1}^{N} a_{n I} s_{n}(y) \int_{0}^{t} e_{n I}(\tau) \int_{L_{x}}^{x} c_{n}(x) \int_{L_{z}}^{z} c_{n}(z) D_{I}(u, y, w, T) d w d u d \tau-\frac{x y \pi}{L_{x} L_{y} L_{z}} \sum_{n=1}^{N} a_{n I} s_{n}(z) \times \\
& \times \int_{0}^{t} e_{n I}(\tau) \int_{L_{x}}^{x} c_{n}(x) \int_{L_{y}}^{y} c_{n}(y) D_{I}(u, v, z, T) d v d u d \tau-\frac{x y z}{L_{x} L_{y} L_{z}} \int_{L_{x}}^{x} \int_{L_{y}}^{y} \int_{L_{z}}^{z}\left[\sum_{n=1}^{N} a_{n I} c_{n}(u) c_{n}(v) c_{n}(w) e_{n I}(t)\right]^{2} \times \\
& \times k_{I, I}(u, v, v, T) d w d v d u-\frac{x y z}{L_{x} L_{y} L_{z}} \int_{L_{x}}^{x} \int_{L_{y}}^{y} \int_{z}^{z} \sum_{n=1}^{N} a_{n I} c_{n}(u) c_{n}(v) c_{n}(w) e_{n I}(t) \sum_{n=1}^{N} a_{n V} c_{n}(u) c_{n}(v) c_{n}(w) \times \\
& \times e_{n V}(t) k_{I, V}(u, v, v, T) d w d v d u+\frac{x y z}{L_{x} L_{y} L_{z}} \int_{L_{x}}^{x} \int_{L_{y}}^{y} \int_{L_{z}}^{z} f_{I}(u, v, w) d w d v d u \\
& \frac{x y z}{\pi^{3}} \sum_{n=1}^{N} \frac{a_{n V}}{n^{3}} s_{n}(x) s_{n}(y) s_{n}(z) e_{n V}(t)=-\frac{y z \pi}{L_{x} L_{y} L_{z}} \sum_{n=1}^{N} a_{n V} \int_{0}^{t} e_{n V}(\tau) \int_{L_{y}}^{y} c_{n}(y) \int_{L_{z}}^{z} c_{n}(z) D_{V}(x, v, w, T) d w d v d \tau \times
\end{aligned}
$$


International Journal of Computational Science, Information Technology and Control Engineering (IJCSITCE) Vol.3, No.3, July 2016

$$
\begin{gathered}
\times s_{n}(x)-\frac{x z \pi}{L_{x} L_{y} L_{z}} \sum_{n=1}^{N} a_{n V} s_{n}(y) \int_{0}^{t} e_{n V}(\tau) \int_{L_{x}}^{x} c_{n}(x) \int_{L_{z}}^{z} c_{n}(z) D_{V}(u, y, w, T) d w d u d \tau-\frac{x y \pi}{L_{x} L_{y} L_{z}} \sum_{n=1}^{N} a_{n V} s_{n}(z) \times \\
\times \int_{0}^{t} e_{n V}(\tau) \int_{L_{x}}^{x} c_{n}(x) \int_{L_{y}}^{y} c_{n}(y) D_{V}(u, v, z, T) d v d u d \tau-\frac{x y z}{L_{x} L_{y} L_{z}} \int_{L_{x} L_{y} L_{z}}^{y} \int_{z}^{z}\left[\sum_{n=1}^{N} a_{n V} c_{n}(u) c_{n}(v) c_{n}(w) e_{n V}(t)\right]^{2} \times \\
\times k_{V, V}(u, v, v, T) d w d v d u-\frac{x y z}{L_{x} L_{y} L_{z}} \int_{L_{x}}^{x} \int_{L_{y}}^{y} \int_{L_{z}}^{z} \sum_{n=1}^{N} a_{n I} c_{n}(u) c_{n}(v) c_{n}(w) e_{n I}(t) \sum_{n=1}^{N} a_{n V} c_{n}(u) c_{n}(v) c_{n}(w) \times \\
\times e_{n V}(t) k_{I, V}(u, v, v, T) d w d v d u+\frac{x y z}{L_{x} L_{y} L_{z}} \int_{L_{x}}^{x} \int_{L_{y} L_{z}}^{y} \int_{V}^{z}(u, v, w) d w d v d u .
\end{gathered}
$$

We determine coefficients $a_{n \rho}$ by using orthogonality condition on the scale of heterostructure. The condition gives us possibility to obtain relations to calculate $a_{n \rho}$ for any quantity $N$ of terms of considered series. In the common case equations for the required coefficients could be written as

$$
\begin{aligned}
& -\frac{L_{x}^{2} L_{y}^{2} L_{z}^{2}}{\pi^{5}} \sum_{n=1}^{N} \frac{a_{n I}}{n^{6}} e_{n I}(t)=-\frac{1}{2 \pi L_{x}} \sum_{n=1}^{N} \frac{a_{n I}}{n^{2}} \int_{0}^{t} e_{n I}(\tau) \int_{0}^{L_{x}}\left[1-c_{n}(2 x)\right] \int_{0}^{L_{y}}\left\{L_{y}+y s_{n}(2 y)+\frac{L_{y}}{2 \pi n}\left[c_{n}(2 y)-1\right]\right\} \times \\
& \times \int_{0}^{L_{z}} D_{I}(x, y, z, T)\left\{z s_{n}(z)+\frac{L_{z}}{2 \pi n}\left[c_{n}(z)-1\right]\right\} d z d y d x d \tau-\frac{1}{2 \pi L_{y}} \sum_{n=1}^{N} \frac{a_{n I}}{n^{2}} \int_{0}^{t} \int_{0}^{L_{x}}\left\{\frac{L_{x}}{\pi n}\left[c_{n}(2 x)-1\right]+L_{x}+\right. \\
& \left.+x s_{n}(2 x)\right\} \int_{0}^{L_{y} L_{z}} D_{0}(x, y, z, T)\left\{L_{z}+z s_{n}(2 z)+\frac{L_{z}}{2 \pi n}\left[c_{n}(2 z)-1\right]\right\} d z\left[1-c_{n}(2 y)\right] d y d x e_{n I}(\tau) d \tau- \\
& -\frac{1}{2 \pi L_{z}} \sum_{n=1}^{N} \frac{a_{n I}}{n^{2}} \int_{0}^{t} e_{n I}(\tau) \int_{0}^{L_{x}}\left\{L_{x}+x s_{n}(2 x)+\frac{L_{x}}{2 \pi n}\left[c_{n}(2 x)-1\right]\right\} \int_{0}^{L_{y}}\left\{L_{y}+y s_{n}(2 y)+\frac{L_{y}}{2 \pi n}\left[c_{n}(2 y)-1\right]\right\} \times \\
& \times \int_{0}^{L_{z}}\left[1-c_{n}(2 z)\right] D_{I}(x, y, z, T) d z d y d x d \tau-\sum_{n=1}^{N} a_{n I}^{2} e_{n I}(2 t) \int_{0}^{L_{x}}\left\{L_{x}+\frac{L_{x}}{2 \pi n}\left[c_{n}(2 x)-1\right]+x s_{n}(2 x)\right\} \times \\
& \times \int_{0}^{L_{y}}\left\{L_{y}+y s_{n}(2 y)+\frac{L_{y}}{2 \pi n}\left[c_{n}(2 y)-1\right]\right\} \int_{0}^{L_{z}} k_{I, I}(x, y, z, T)\left\{L_{z}+\frac{L_{z}}{2 \pi n}\left[c_{n}(2 z)-1\right]+z s_{n}(2 z)\right\} d z d y d x- \\
& -\sum_{n=1}^{N} a_{n I} a_{n V} e_{n I}(t) e_{n V}(t) \int_{0}^{L_{x}}\left\{L_{x}+x s_{n}(2 x)+\frac{L_{x}}{2 \pi n}\left[c_{n}(2 x)-1\right]\right\} \int_{0}^{L_{y}}\left\{L_{y}+y s_{n}(2 y)+\frac{L_{y}}{2 \pi n}\left[c_{n}(2 y)-1\right]\right\} \times \\
& \times \int_{0}^{L_{z}} k_{I, V}(x, y, z, T)\left\{L_{z}+z s_{n}(2 z)+\frac{L_{z}}{2 \pi n}\left[c_{n}(2 z)-1\right]\right\} d z d y d x+\sum_{n=1}^{N} \int_{0}^{L_{x}}\left\{x s_{n}(x)+\frac{L_{x}}{\pi n}\left[c_{n}(x)-1\right]\right\} \times \\
& \times \int_{0}^{L_{y}}\left\{y s_{n}(y)+\frac{L_{y}}{\pi n}\left[c_{n}(y)-1\right]\right\} \int_{0}^{L_{z}} f_{I}(x, y, z, T)\left\{L_{z}+z s_{n}(2 z)+\frac{L_{z}}{2 \pi n}\left[c_{n}(2 z)-1\right]\right\} d z d y d x \\
& -\frac{L_{x}^{2} L_{y}^{2} L_{z}^{2}}{\pi^{5}} \sum_{n=1}^{N} \frac{a_{n V}}{n^{6}} e_{n V}(t)=-\frac{1}{2 \pi L_{x}} \sum_{n=1}^{N} \frac{a_{n V}}{n^{2}} \int_{0}^{t} e_{n V}(\tau) \int_{0}^{L_{x}}\left[1-c_{n}(2 x)\right] \int_{0}^{L_{y}}\left\{L_{y}+y s_{n}(2 y)+\frac{L_{y}}{2 \pi n}\left[c_{n}(2 y)-1\right]\right\} \times \\
& \times \int_{0}^{L_{z}} D_{V}(x, y, z, T)\left\{z s_{n}(z)+\frac{L_{z}}{2 \pi n}\left[c_{n}(z)-1\right]\right\} d z d y d x d \tau-\frac{1}{2 \pi L_{y}} \sum_{n=1}^{N} \frac{a_{n V}}{n^{2}} \int_{0}^{t} \int_{0}^{L_{x}}\left\{\frac{L_{x}}{\pi n}\left[c_{n}(2 x)-1\right]+L_{x}+\right. \\
& \left.+x s_{n}(2 x)\right\} \int_{0}^{L_{y}} \int_{0}^{L_{z}} D_{V}(x, y, z, T)\left\{L_{z}+z s_{n}(2 z)+\frac{L_{z}}{2 \pi n}\left[c_{n}(2 z)-1\right]\right\} d z\left[1-c_{n}(2 y)\right] d y d x e_{n V}(\tau) d \tau- \\
& -\frac{1}{2 \pi L_{z}} \sum_{n=1}^{N} \frac{a_{n V}}{n^{2}} \int_{0}^{t} e_{n V}(\tau) \int_{0}^{L_{x}}\left\{L_{x}+x s_{n}(2 x)+\frac{L_{x}}{2 \pi n}\left[c_{n}(2 x)-1\right]\right\} \int_{0}^{L_{y}}\left\{L_{y}+y s_{n}(2 y)+\frac{L_{y}}{2 \pi n}\left[c_{n}(2 y)-1\right]\right\} \times
\end{aligned}
$$



(IJCSITCE) Vol.3, No.3, July 2016

$$
\begin{aligned}
& \times \int_{0}^{L_{z}}\left[1-c_{n}(2 z)\right] D_{V}(x, y, z, T) d z d y d x d \tau-\sum_{n=1}^{N} a_{n V}^{2} e_{n V}(2 t) \int_{0}^{L_{x}}\left\{L_{x}+\frac{L_{x}}{2 \pi n}\left[c_{n}(2 x)-1\right]+x s_{n}(2 x)\right\} \times \\
& \times \int_{0}^{L_{y}}\left\{L_{y}+y s_{n}(2 y)+\frac{L_{y}}{2 \pi n}\left[c_{n}(2 y)-1\right]\right\} \int_{0}^{L_{z}} k_{V, V}(x, y, z, T)\left\{L_{z}+\frac{L_{z}}{2 \pi n}\left[c_{n}(2 z)-1\right]+z s_{n}(2 z)\right\} d z d y d x- \\
& -\sum_{n=1}^{N} a_{n I} a_{n V} e_{n I}(t) e_{n V}(t) \int_{0}^{L_{x}}\left\{L_{x}+x s_{n}(2 x)+\frac{L_{x}}{2 \pi n}\left[c_{n}(2 x)-1\right]\right\} \int_{0}^{L_{y}}\left\{L_{y}+y s_{n}(2 y)+\frac{L_{y}}{2 \pi n}\left[c_{n}(2 y)-1\right]\right\} \times \\
& \times \int_{0}^{L_{z}} k_{I, V}(x, y, z, T)\left\{L_{z}+z s_{n}(2 z)+\frac{L_{z}}{2 \pi n}\left[c_{n}(2 z)-1\right]\right\} d z d y d x+\sum_{n=1}^{N} \int_{0}^{L_{x}}\left\{x s_{n}(x)+\frac{L_{x}}{\pi n}\left[c_{n}(x)-1\right]\right\} \times \\
& \quad \times \int_{0}^{L_{y}}\left\{y s_{n}(y)+\frac{L_{y}}{\pi n}\left[c_{n}(y)-1\right]\right\} \int_{0}^{L_{z}} f_{V}(x, y, z, T)\left\{L_{z}+z s_{n}(2 z)+\frac{L_{z}}{2 \pi n}\left[c_{n}(2 z)-1\right]\right\} d z d y d x .
\end{aligned}
$$

In the final form relations for required parameters could be written as

$$
a_{n I}=-\frac{b_{3}+A}{4 b_{4}} \pm \sqrt{\frac{\left(b_{3}+A\right)^{2}}{4}-4 b_{4}\left(y+\frac{b_{3} y-\gamma_{n V} \lambda_{n I}^{2}}{A}\right)}, a_{n V}=-\frac{\gamma_{n I} a_{n I}^{2}+\delta_{n I} a_{n I}+\lambda_{n I}}{\chi_{n I} a_{n I}},
$$

where $\gamma_{n \rho}=e_{n \rho}(2 t) \iint_{0}^{L_{x} L_{y} L_{z}} \int_{0} k_{\rho, \rho}(x, y, z, T)\left\{L_{x}+x s_{n}(2 x)+\frac{L_{x}}{2 \pi n}\left[c_{n}(2 x)-1\right]\right\}\left\{y s_{n}(2 y)+L_{y}+\frac{L_{y}}{2 \pi n} \times\right.$ $\left.\times\left[c_{n}(2 y)-1\right]\right\}\left\{L_{z}+z s_{n}(2 z)+\frac{L_{z}}{2 \pi n}\left[c_{n}(2 z)-1\right]\right\} d z d y d x, \delta_{n \rho}=\frac{1}{2 \pi L_{x} n^{2}} \int_{0}^{t} e_{n \rho}(\tau) \int_{0}^{L_{x} L_{y}}\left\{y s_{0}\{y)+\right.$ $\left.+\frac{L_{y}}{2 \pi n}\left[c_{n}(y)-1\right]\right\} \int_{0}^{L_{z}}\left\{z s_{n}(z)+\frac{L_{z}}{2 \pi n}\left[c_{n}(z)-1\right]\right\} D_{\rho}(x, y, z, T) d z d y\left[1-c_{n}(2 x)\right] d x d \tau+\frac{1}{2 \pi L_{y}} \times$ $\times \frac{1}{2 n^{2}} \int_{0}^{t} e_{n \rho}(\tau) \int_{0}^{L_{x}}\left\{L_{x}+x s_{n}(2 x)+\frac{L_{x}}{\pi n}\left[c_{n}(2 x)-1\right]\right\} \int_{0}^{L_{y}}\left[1-c_{n}(2 y)\right] \int_{0}^{L_{z}} D_{\rho}(x, y, z, T)\left\{\frac{L_{z}}{2 \pi n}\left[c_{n}(2 z)-1\right]+\right.$ $\left.+z s_{n}(2 z)+L_{z}\right\} d z d y d x d \tau+\frac{1}{2 \pi L_{z} n^{2}} \int_{0}^{t} e_{n \rho}(\tau) \int_{0}^{L_{x}}\left\{x s_{n}(2 x)+L_{x}+\frac{L_{x}}{\pi n}\left[c_{n}(2 x)-1\right]\right\} \int_{0}^{L_{y}}\left\{y s_{n}(y)+\right.$ $+L_{y}+\frac{L_{y}}{2 \pi n}\left[c_{n}(y)-1\right] \int_{0}^{L_{z}}\left[1-c_{n}(2 z)\right] D_{\rho}(x, y, z, T) d z d y d x d \tau-\frac{L_{x}^{2} L_{y}^{2} L_{z}^{2}}{\pi^{5} n^{6}} e_{n \rho}(t), \chi_{n I V}=e_{n I}(t) \times$ $\times e_{n V}(t) \int_{0}^{L_{x}}\left\{x s_{n}(x)+\frac{L_{x}}{\pi n}\left[c_{n}(x)-1\right]\right\} \int_{0}^{L_{y}}\left\{L_{y}+\frac{L_{y}}{2 \pi n}\left[c_{n}(2 y)-1\right]+y s_{n}(2 y)\right\} \int_{0}^{L_{z}} k_{I, V}(x, y, z, T)\left\{L_{z}+z s_{n}(2 z)+\right.$ $\left.+\frac{L_{z}}{2 \pi n}\left[c_{n}(2 z)-1\right]\right\} d z d y d x, \lambda_{n \rho}=\int_{0}^{L_{x}}\left\{x s_{n}(x)+\frac{L_{x}}{\pi n}\left[c_{n}(x)-1\right] \int_{0}^{L_{y}}\left\{y s_{n}(y)+\frac{L_{y}}{\pi n}\left[c_{n}(y)-1\right]\right\} \int_{0}^{L_{z}}\{z \times\right.$ $\left.\times s_{n}(z)+\frac{L_{z}}{\pi n}\left[c_{n}(z)-1\right]\right\} f_{\rho}(x, y, z, T) d z d y d x, b_{4}=\gamma_{n V} \gamma_{n I}^{2}-\gamma_{n I} \chi_{n I}^{2}, b_{3}=2 \gamma_{n V} \gamma_{n I} \delta_{n I}-\delta_{n I} \chi_{n I}^{2}-$ $-\delta_{n V} \chi_{n I} \gamma_{n I}, b_{2}=\gamma_{n V} \delta_{n I}^{2}+2 \lambda_{n I} \gamma_{n V} \gamma_{n I}-\delta_{n V} \chi_{n I} \delta_{n I}+\left(\lambda_{n V}-\lambda_{n I}\right) \chi_{n I}^{2}, b_{1}=2 \lambda_{n I} \gamma_{n V} \delta_{n I}-\delta_{n V} \chi_{n I} \lambda_{n I}$, $A=\sqrt{8 y+b_{3}^{2}-4 b_{2}}, p=\frac{3 b_{2} b_{4}-b_{3}^{2}}{9 b_{4}^{2}}, q=\frac{2 b_{3}^{3}-9 b_{2} b_{3}+27 b_{1} b_{4}^{2}}{54 b_{4}^{3}}, y=\sqrt[3]{\sqrt{q^{2}+p^{3}}-q}-\sqrt[3]{\sqrt{q^{2}+p^{3}}+q}-$ $-b_{3} / 3 b_{4}$. 
We determine spatio-temporal distributions of concentrations of complexes of radiation defects in the following form

$$
\Phi_{\rho 0}(x, y, z, t)=\sum_{n=1}^{N} a_{n \Phi \rho} c_{n}(x) c_{n}(y) c_{n}(z) e_{n \rho}(t),
$$

where $a_{n \Phi \rho}$ are not yet known coefficients. Let us previously transform the Eqs.(6) to the following integro-differential form

$$
\begin{aligned}
& \frac{x y z}{L_{x} L_{y} L_{z}} \int_{L_{x} L_{y} L_{z}}^{x} \int_{z}^{z} \Phi_{I}(u, v, w, t) d w d v d u=\frac{y z}{L_{y} L_{z}} \int_{0}^{t} \int_{L_{y} L_{z}}^{y} \int_{\Phi_{I}}^{z} D_{I}(x, v, w, T) \frac{\partial \Phi_{I}(x, v, w, \tau)}{\partial x} d w d v d \tau+ \\
& +\frac{x z}{L_{x} L_{z}} \int_{0}^{t} \int_{L_{x} L_{z}}^{x} \int_{\Phi}^{z} D_{\Phi}(u, y, w, T) \frac{\partial \Phi_{I}(u, y, w, \tau)}{\partial y} d w d u d \tau+\int_{0}^{t} \int_{L_{x} L_{y}}^{x} D_{\Phi I}(u, v, z, T) \frac{\partial \Phi_{I}(u, v, z, \tau)}{\partial z} d v d u d \tau \times \\
& \times \frac{x y}{L_{x} L_{y}}+\frac{x y z}{L_{x} L_{y} L_{z}} \int_{L_{x}}^{x} \int_{L_{y} L_{z}}^{y} \int_{I, I}^{z} k_{I,}(u, v, w, T) I^{2}(u, v, w, \tau) d w d v d u-\frac{x y z}{L_{x} L_{y} L_{z}} \int_{L_{x} L_{y} L_{z}}^{x} \int_{I}^{z} k_{I}(u, v, w, T) \times \\
& \times I(u, v, w, \tau) d w d v d u+\frac{x y z}{L_{x} L_{y} L_{z}} \int_{L_{x}}^{x} \int_{y}^{y} \int_{z}^{z} f_{\Phi I}(u, v, w) d w d v d u \\
& \frac{x y z}{L_{x} L_{y} L_{z}} \int_{L_{x} L_{y} L_{z}}^{x} \int_{V}^{z} \Phi_{V}(u, v, w, t) d w d v d u=\frac{y z}{L_{y} L_{z}} \int_{0}^{t} \int_{L_{y} L_{z}}^{y} \int_{\Phi_{V}}^{z} D_{\infty}(x, v, w, T) \frac{\partial \Phi_{V}(x, v, w, \tau)}{\partial x} d w d v d \tau+ \\
& +\frac{x z}{L_{x} L_{z}} \int_{0}^{t} \int_{L_{x}}^{x} \int_{L_{z}}^{z} D_{\Phi V}(u, y, w, T) \frac{\partial \Phi_{V}(u, y, w, \tau)}{\partial y} d w d u d \tau+\int_{0}^{t} \int_{L_{x} L_{y}}^{y} D_{\Phi V}(u, v, z, T) \frac{\partial \Phi_{V}(u, v, z, \tau)}{\partial z} d v d u d \tau \times \\
& \times \frac{x y}{L_{x} L_{y}}+\frac{x y z}{L_{x} L_{y} L_{z}} \int_{L_{x} L_{y} L_{z}}^{x} \int_{V, V}^{z} k_{V}(u, v, w, T) V^{2}(u, v, w, \tau) d w d v d u-\frac{x y z}{L_{x} L_{y} L_{z}} \int_{L_{x}}^{x} \int_{L_{y} L_{z}}^{y} k_{V}(u, v, w, T) \times \\
& \times V(u, v, w, \tau) d w d v d u+\frac{x y z}{L_{x} L_{y} L_{z}} \int_{L_{x} L_{y} L_{z}}^{x} \int_{\Phi V}^{z} f_{\Phi V}(u, v, w) d w d v d u .
\end{aligned}
$$

Substitution of the previously considered series in the Eqs.(6a) leads to the following form

$$
\begin{aligned}
& -x y z \sum_{n=1}^{N} \frac{a_{n \Phi I}}{\pi^{3} n^{3}} s_{n}(x) s_{n}(y) s_{n}(z) e_{n I}(t)=-\frac{y z \pi}{L_{x} L_{y} L_{z}} \sum_{n=1}^{N} n a_{n \Phi I} s_{n}(x) e_{n I}(t) \int_{0}^{t} \int_{L_{y}}^{y} c_{n}(v) \int_{L_{z}}^{z} D_{\Phi I}(x, v, w, T) \times \\
& \times c_{n}(w) d w d v d \tau-\frac{x z \pi}{L_{x} L_{y} L_{z}} \sum_{n=1}^{N} a_{n \Phi I} n s_{n}(y) e_{n \Phi I}(t) \int_{0}^{t} \int_{L_{x} L_{z}}^{x} \int_{n}^{z} c_{n}(u) c_{n}(w) D_{\Phi I}(u, v, w, T) d w d u d \tau- \\
& -\frac{x y \pi}{L_{x} L_{y} L_{z}} \sum_{n=1}^{N} n a_{n \Phi I} s_{n}(z) e_{n \Phi I}(t) \int_{0}^{t} \int_{L_{x} L_{y}}^{x} \int_{n}^{y} c_{n}(u) c_{n}(v) D_{\Phi I}(u, v, z, T) d v d u d \tau+\int_{L_{x} L_{y}}^{x} \int_{L_{z}}^{z} k_{I, I}(u, v, w, T) \times \\
& \times I^{2}(u, v, w, \tau) d w d v d u \frac{x y z}{L_{x} L_{y} L_{z}}-\frac{x y z}{L_{x} L_{y} L_{z}} \int_{L_{x}}^{x} \int_{y}^{y} \int_{L_{z}}^{z} k_{I}(u, v, w, T) I(u, v, w, \tau) d w d v d u+\frac{x y z}{L_{x} L_{y} L_{z}} \times \\
& \times \int_{L_{x}}^{x} \int_{L_{y} L_{z}}^{y} f_{\Phi I}^{z}(u, v, w) d w d v d u \\
& -x y z \sum_{n=1}^{N} \frac{a_{n \Phi V}}{\pi^{3} n^{3}} s_{n}(x) s_{n}(y) s_{n}(z) e_{n V}(t)=-\frac{y z \pi}{L_{x} L_{y} L_{z}} \sum_{n=1}^{N} n a_{n \Phi V} s_{n}(x) e_{n V}(t) \int_{0}^{t} \int_{L_{y}}^{y} c_{n}(v) \int_{L_{z}}^{z} D_{\Phi V}(x, v, w, T) \times \\
& \times c_{n}(w) d w d v d \tau-\frac{x z \pi}{L_{x} L_{y} L_{z}} \sum_{n=1}^{N} a_{n \Phi V} n s_{n}(y) e_{n \Phi V}(t) \int_{0}^{t} \int_{L_{x}}^{x} \int_{z}^{z} c_{n}(u) c_{n}(w) D_{\Phi V}(u, v, w, T) d w d u d \tau-
\end{aligned}
$$


International Journal of Computational Science, Information Technology and Control Engineering (IJCSITCE) Vol.3, No.3, July 2016

$-\frac{x y \pi}{L_{x} L_{y} L_{z}} \sum_{n=1}^{N} n a_{n \Phi V} s_{n}(z) e_{n \Phi V}(t) \int_{0}^{t} \int_{L_{x} L_{y}}^{x} c_{n}^{y}(u) c_{n}(v) D_{\Phi V}(u, v, z, T) d v d u d \tau+\int_{L_{x} L_{y} L_{z}}^{x} \int_{V, V}^{z} k_{V, V}(u, v, w, T) \times$ $\times V^{2}(u, v, w, \tau) d w d v d u \frac{x y z}{L_{x} L_{y} L_{z}}-\frac{x y z}{L_{x} L_{y} L_{z}} \int_{L_{x} L_{y} L_{z}}^{x} \int_{v}^{z} k_{V}(u, v, w, T) V(u, v, w, \tau) d w d v d u+\frac{x y z}{L_{x} L_{y} L_{z}} \times$ $\times \int_{L_{x}}^{x} \int_{L_{y} L_{z}}^{y} f_{\Phi V}^{z}(u, v, w) d w d v d u$

We determine coefficients $a_{n}$ o by using orthogonality condition on the scale of heterostructure. The condition gives us possibility to obtain relations to calculate $a_{n \Phi \rho}$ for any quantity $N$ of terms of considered series. In the common case equations for the required coefficients could be written as

$$
\begin{aligned}
& -\frac{L_{x}^{2} L_{y}^{2} L_{z}^{2}}{\pi^{5}} \sum_{n=1}^{N} \frac{a_{n \Phi I}}{n^{6}} e_{n \Phi I}(t)=-\frac{1}{2 \pi L_{x}} \sum_{n=1}^{N} \frac{a_{n \Phi I}}{n^{2}} \int_{0}^{t} \int_{0}^{L_{x}}\left[1-c_{n}(2 x)\right] \int_{0}^{L_{y}}\left\{L_{y}+y s_{n}(2 y)+\frac{L_{y}}{2 \pi n}\left[c_{n}(2 y)-1\right]\right\} \times \\
& \times \int_{0}^{L_{z}} D_{\Phi I}(x, y, z, T)\left\{z s_{n}(z)+\frac{L_{z}}{2 \pi n}\left[c_{n}(z)-1\right]\right\} d z d y d x e_{n \Phi I}(\tau) d \tau-\frac{1}{2 \pi} \sum_{n=1}^{N} \frac{a_{n \Phi I}}{n^{2} L_{y}} \int_{0}^{t} \int_{0}^{L_{x}}\left\{x s_{n}(2 x)+L_{x}+\right. \\
& \left.+L_{x} \frac{c_{n}(2 x)-1}{2 \pi n}\right\} \int_{0}^{L_{y}}\left[1-c_{n}(2 y)\right] \int_{0}^{L_{z}} D_{\Phi I}(x, y, z, T)\left\{z s_{n}(z)+\frac{L_{z}}{2 \pi n}\left[c_{n}(z)-1\right]\right\} d z d y d x e_{n \Phi I}(\tau) d \tau-\frac{1}{\pi L_{x}} \times \\
& \times \sum_{n=1}^{N} \frac{a_{n \Phi I}}{2 n^{2}} \int_{0}^{t} e_{n \Phi I}(\tau) \int_{0}^{L_{x}}\left\{x s_{n}(x)+\frac{L_{x}}{2 \pi n}\left[c_{n}(x)-1\right]\right\} \int_{0}^{L_{y}}\left\{y s_{n}(2 y)+\frac{L_{y}}{2 \pi n}\left[c_{n}(2 y)-1\right]+L_{y}\right\} \int_{0}^{L_{z}} D_{\Phi I}(x, y, z, T) \times \\
& \times\left[1-c_{n}(2 y)\right] d z d y d x d \tau+\sum_{n=1}^{N} \int_{0}^{t} e_{n \Phi I}(\tau) \int_{0}^{L_{x}}\left\{\frac{L_{x}}{2 \pi n}\left[c_{n}(x)-1\right]+x s_{n}(x)\right\} \int_{0}^{L_{y}}\left\{y s_{n}(y)+\frac{L_{y}}{2 \pi n}\left[c_{n}(y)-1\right]\right\} \times \\
& \times \frac{a_{n \Phi I}}{n^{3} \pi^{3}} \int_{0}^{L_{z}} I^{2}(x, y, z, t) k_{I, I}(x, y, z, T)\left\{\frac{L_{z}}{2 \pi n}\left[c_{n}(z)-1\right]+z s_{n}(z)\right\} d z d y d x-\sum_{n=10}^{N} \int_{n \Phi I}^{t} e_{n}(\tau) \int_{0}^{L_{x}}\left\{x s_{n}(x)+\right. \\
& \left.+\frac{L_{x}}{2 \pi n}\left[c_{n}(x)-1\right]\right\} \frac{a_{n \Phi I}}{n^{3} \pi^{3}} \int_{0}^{L_{y}}\left\{\frac{L_{y}}{2 \pi n}\left[c_{n}(y)-1\right]+y s_{n}(y)\right\} \int_{0}^{L_{z}} k_{I}(x, y, z, T) I(x, y, z, t)\left\{\frac{L_{z}}{2 \pi n}\left[c_{n}(z)-1\right]+\right. \\
& \left.+z s_{n}(z)\right\} d z d y d x+\sum_{n=1}^{N} \frac{a_{n \Phi I}}{\pi^{3} n^{3}} \int_{0}^{t} e_{n \Phi I}(\tau) \int_{0}^{L_{x}}\left\{x s_{n}(x)+\frac{L_{x}}{2 \pi n}\left[c_{n}(x)-1\right] \int_{0}^{L_{y}}\left\{y s_{n}(y)+\frac{L_{y}}{2 \pi n}\left[c_{n}(y)-1\right]\right\} \times\right. \\
& \times \int_{0}^{L_{z}}\left\{\frac{L_{z}}{2 \pi n}\left[c_{n}(z)-1\right]+z s_{n}(z)\right\} f_{\Phi I}(x, y, z) d z d y d x \\
& -\frac{L_{x}^{2} L_{y}^{2} L_{z}^{2}}{\pi^{5}} \sum_{n=1}^{N} \frac{a_{n \Phi V}}{n^{6}} e_{n \Phi V}(t)=-\frac{1}{2 \pi L_{x}} \sum_{n=1}^{N} \frac{a_{n \Phi V}}{n^{2}} \int_{0}^{t} \int_{0}^{L_{x}}\left[1-c_{n}(2 x)\right] \int_{0}^{L_{y}}\left\{L_{y}+y s_{n}(2 y)+\frac{L_{y}}{2 \pi n}\left[c_{n}(2 y)-1\right]\right\} \times \\
& \times \int_{0}^{L_{z}} D_{\Phi V}(x, y, z, T)\left\{z s_{n}(z)+\frac{L_{z}}{2 \pi n}\left[c_{n}(z)-1\right]\right\} d z d y d x e_{n \Phi V}(\tau) d \tau-\frac{1}{2 \pi} \sum_{n=1}^{N} \frac{a_{n \Phi V}}{n^{2} L_{y}} \int_{0}^{t} \int_{0}^{L_{x}}\left\{x s_{n}(2 x)+L_{x}+\right. \\
& +L_{x} \frac{c_{n}(2 x)-1}{2 \pi n} \int_{0}^{L_{y}}\left[1-c_{n}(2 y)\right] \int_{0}^{L_{z}} D_{\Phi V}(x, y, z, T)\left\{z s_{n}(z)+\frac{L_{z}}{2 \pi n}\left[c_{n}(z)-1\right]\right\} d z d y d x e_{n \Phi V}(\tau) d \tau-\frac{1}{\pi L_{x}} \times \\
& \times \sum_{n=1}^{N} \frac{a_{n \Phi V}}{2 n^{2}} \int_{0}^{t} e_{n \Phi V}(\tau) \int_{0}^{L_{x}}\left\{x s_{n}(x)+\frac{L_{x}}{2 \pi n}\left[c_{n}(x)-1\right]\right\} \int_{0}^{L_{y}}\left\{y s_{n}(2 y)+\frac{L_{y}}{2 \pi n}\left[c_{n}(2 y)-1\right]+L_{y}\right\} \int_{0}^{L_{z}} D_{\Phi V}(x, y, z, T) \times
\end{aligned}
$$




$$
\begin{aligned}
& \times\left[1-c_{n}(2 y)\right] d z d y d x d \tau+\sum_{n=1}^{N} \int_{0}^{t} e_{n \Phi V}(\tau) \int_{0}^{L_{x}}\left\{\frac{L_{x}}{2 \pi n}\left[c_{n}(x)-1\right]+x s_{n}(x)\right\} \int_{0}^{L_{y}}\left\{y s_{n}(y)+\frac{L_{y}}{2 \pi n}\left[c_{n}(y)-1\right]\right\} \times \\
& \times \frac{a_{n \Phi V}}{n^{3} \pi^{3}} \int_{0}^{L_{z}} V^{2}(x, y, z, t) k_{V, V}(x, y, z, T)\left\{\frac{L_{z}}{2 \pi n}\left[c_{n}(z)-1\right]+z s_{n}(z)\right\} d z d y d x-\sum_{n=10}^{N} \int_{n \Phi V}^{t}(\tau) \int_{0}^{L_{x}}\left\{x s_{n}(x)+\right. \\
& \left.+\frac{L_{x}}{2 \pi n}\left[c_{n}(x)-1\right]\right\} \frac{a_{n \Phi V}}{n^{3} \pi^{3}} \int_{0}^{L_{y}}\left\{\frac{L_{y}}{2 \pi n}\left[c_{n}(y)-1\right]+y s_{n}(y)\right\} \int_{0}^{L_{z}} k_{V}(x, y, z, T) V(x, y, z, t)\left\{\frac{L_{z}}{2 \pi n}\left[c_{n}(z)-1\right]+\right. \\
& \left.+z s_{n}(z)\right\} d z d y d x+\sum_{n=1}^{N} \frac{a_{n \Phi V}}{\pi^{3} n^{3}} \int_{0}^{t} e_{n \Phi V}(\tau) \int_{0}^{L_{x}}\left\{x s_{n}(x)+\frac{L_{x}}{2 \pi n}\left[c_{n}(x)-1\right]\right\} \int_{0}^{L_{y}}\left\{y s_{n}(y)+\frac{L_{y}}{2 \pi n}\left[c_{n}(y)-1\right]\right\} \times \\
& \times \int_{0}^{L_{z}}\left\{\frac{L_{z}}{2 \pi n}\left[c_{n}(z)-1\right]+z s_{n}(z)\right\} f_{\Phi V}(x, y, z) d z d y d x .
\end{aligned}
$$

\section{DISCUSSION}

In this section we analyzed distribution of concentration of dopant, infused (see Fig. $2 a$ ) or implanted (see Fig. 2b) into epitaxial layer. Annealing time is the same for all curves of these figures. Increasing of number of curves corresponds to increasing of difference between values of dopant diffusion coefficients in layers of hetero structure. The figures show, that presents of interface between layers of hetero structure gives a possibility to increase absolute value of gradient of concentration of dopant in direction, which is perpendicular to the considered interface. A consequence of this result is decreasing of the dimensions of transistors included in the multivibrator. At the same time with increasing of the considered gradient homogeneity of concentration of dopant in enriched area.

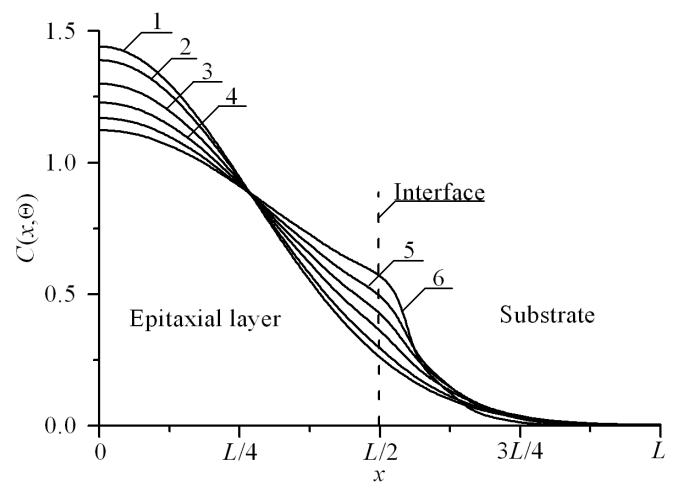

Fig.2a. Distributions of concentration of infused dopant in heterostructure from Figs. 1 in direction, which is perpendicular to interface between epitaxial layer substrate. Increasing of number of curve corresponds to increasing of difference between values of dopant diffusion coefficient in layers of heterostructure under condition, when value of dopant diffusion coefficient in epitaxial layer is larger, than value of dopant diffusion coefficient in substrate

To estimate annealing time it is necessary to estimate decreasing of absolute value of gradient of concentration of dopant near interface between substrate and epitaxial layer with increasing of annealing time. Decreasing of annealing time leads to manufacturing more inhomogenous distribution of concentration of dopant (see Fig. $3 a$ for diffusion type of doping and Fig. $3 b$ for ion type of doping). We determine compromise value of annealing time framework recently introduced criterion [14-20]. Frame-work the criterion we approximate real distribution of concentration of dopant by idealized step-wise function $\psi(x, y, z)$. Further we determine the required annealing time by minimization of mean-square error 


$$
U=\frac{1}{L_{x} L_{y} L_{z}} \int_{0}^{L_{x}} \int_{0}^{L_{y} L_{z}} \int_{0}^{0}[C(x, y, z, \Theta)-\psi(x, y, z)] d z d y d x .
$$

Dependences of optimal annealing time are shown on Figs. 4. It should be noted, that radiation defects, generated during ion implantation, should be annealed. In the ideal case after the annealing the implanted dopant should achieve the interface between layers of heterostructure. If the dopant has no enough time to achieve the interface, it is attracted an interest to make additional annealing. The Fig. $4 b$ shows dependences of additional annealing time. Necessity of annealing of radiation defects leads to smaller value of optimal annealing time of dopant in comparison with optimal annealing time of dopant during diffusion type of doping. Diffusion type of doping did not leads to radiation damage of materials. Ion type of doping gives us possibility to decrease mismatch-induced stress in heterostructure [20].

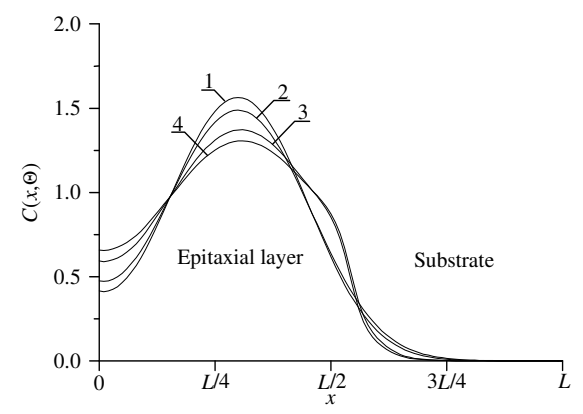

Fig. $2 b$. Distributions of concentration of implanted dopant in heterostructure from Figs. 1 in direction, which is perpendicular to interface between epitaxial layer substrate. Curves 1 and 3 corresponds to annealing time $\Theta=0.0048\left(L_{x}^{2}+L_{y}{ }^{2}+L_{z}^{2}\right) / D_{0}$. Curves 2 and 4 corresponds to annealing time $\Theta=0.0057\left(L_{x}{ }^{2}+\right.$ $\left.L_{y}{ }^{2}+L_{z}^{2}\right) / D_{0}$. Curves 1 and 2 corresponds to homogenous sample. Curves 3 and 4 corresponds to heterostructure under condition, when value of dopant diffusion coefficient in epitaxial layer is larger, than value of dopant diffusion coefficient in substrate

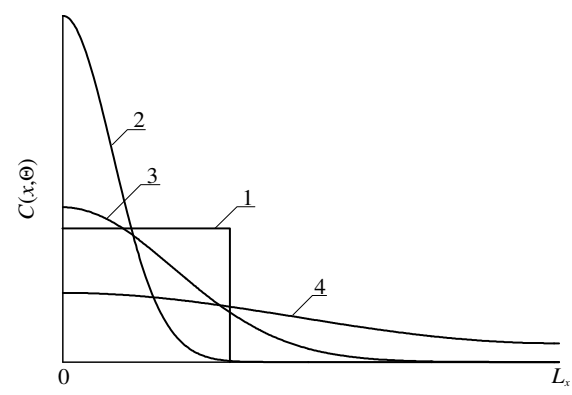

Fig. 3a. Spatial distributions of dopant in heterostructure after dopant infusion. Curve 1 is idealized distribution of dopant. Curves 2-4 are real distributions of dopant for different values of annealing time. Increasing of number of curve corresponds to increasing of annealing time

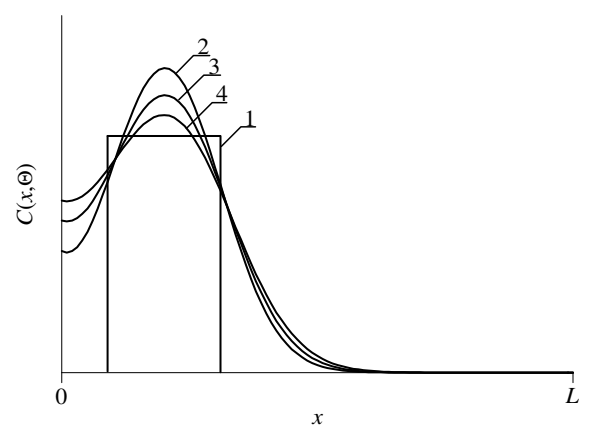


Fig.3b. Spatial distributions of dopant in heterostructure after ion implantation. Curve 1 is idealized distribution of dopant. Curves 2-4 are real distributions of dopant for different values of annealing time. Increasing of number of curve corresponds to increasing of annealing time

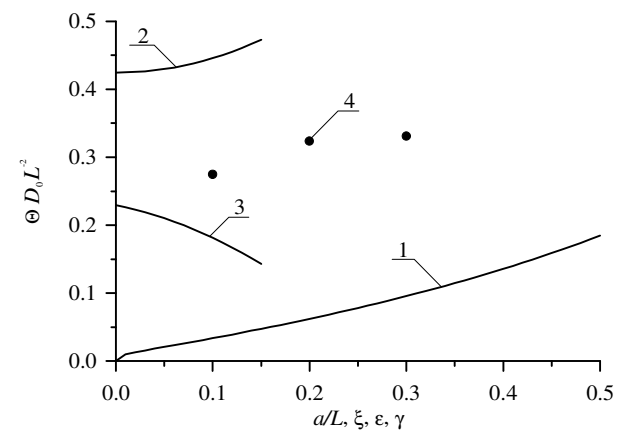

Fig.4a. Optimal annealing time of infused dopant as dependences of several parameters. Curve 1 is the dependence of the considered annealing time on dimensionless thickness of epitaxial layer $a / L$ and $\xi=\gamma=$ 0 for equal to each other values of dopant diffusion coefficient in all parts of heterostructure. Curve 2 is the dependence of the considered annealing time on the parameter $\varepsilon$ for $a / L=1 / 2$ and $\xi=\gamma=0$. Curve 3 is the dependence of the considered annealing time on the parameter $\xi$ for $a / L=1 / 2$ and $\varepsilon=\gamma=0$. Curve 4 is the dependence of the considered annealing time on parameter $\gamma$ for $a / L=1 / 2$ and $\varepsilon=\xi=0$

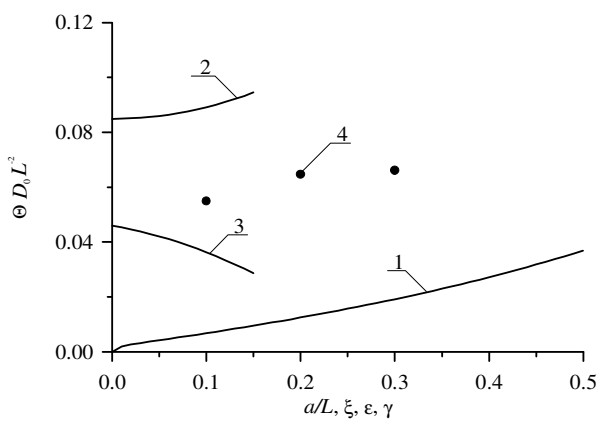

Fig. $4 b$. Optimal annealing time of implanted dopant as dependences of several parameters. Curve 1 is the dependence of the considered annealing time on dimensionless thickness of epitaxial layer $a / L$ and $\xi=\gamma=0$ for equal to each other values of dopant diffusion coefficient in all parts of heterostructure. Curve 2 is the dependence of the considered annealing time on the parameter $\varepsilon$ for $a / L=1 / 2$ and $\xi=\gamma=0$. Curve 3 is the dependence of the considered annealing time on the parameter $\xi$ for $a / L=1 / 2$ and $\varepsilon=\gamma=0$. Curve 4 is the dependence of the considered annealing time on parameter $\gamma$ for $a / L=1 / 2$ and $\varepsilon=\xi=0$

\section{CONCLUSIONS}


In this paper prognosis of time varying of spatial distributions of concentrations of infused and implanted dopants during manufacturing a transistor multivibrator has been done. Several recommendations to optimize manufacture the heterotransistors have been formulated. Analytical approach to prognosis diffusion and ion types of doping has been introduced. The approach gives a possibility to take into account variation in space and time parameters of doped material and at the same time nonlinearity of mass and heat transport.

\section{ACKNOWLEDGEMENTS}

This work is supported by the agreement of August 27, 2013 № 02.B.49.21.0003 between The Ministry of education and science of the Russian Federation and Lobachevsky State University of Nizhni Novgorod, educational fellowship for scientific research of Government of Russian, educational fellowship for scientific research of Government of Nizhny Novgorod region of Russia and educational fellowship for scientific research of Nizhny Novgorod State University of Architecture and Civil Engineering.

\section{REFERENCES}

[1] Z. Ramezani, A.A. Orouji. A silicon-on-insulator metal semiconductor field-effect transistor with an L-shaped buried oxide for high output-power density. Mat. Sci. Sem. Proc. Vol. 19. P. 124-129 (2014).

[2] Ch. Dong, J. Shi, J. Wu, Y. Chen, D. Zhou, Z. Hu, H. Xie, R. Zhan, Zh. Zou. Improvements in passivation effect of amorphous InGaZnO thin film transistors. Mat. Sci. Sem. Proc. Vol. 20. P. 711 (2014).

[3] D. Fathi, B. Forouzandeh. Accurate analysis of global interconnects in nano-fpgas. Nano. Vol. 4 (3). P. 171-176 (2009).

[4] D. Fathi, B. Forouzandeh, N. Masoumi. New enhanced noise analysis in active mixers in nanoscale technologies. Nano. Vol. 4 (4). P. 233-238 (2009).

[5] A.O. Ageev, A.E. Belyaev, N.S. Boltovets, V.N. Ivanov, R.V. Konakova, Ya.Ya. Kudrik, P.M. Litvin, V.V. Milenin, A.V. Sachenko. Au-TiBx-n-6H-SiC Schottky barrier diodes: Specific features of charge transport in rectifying and nonrectifying contacts. Semiconductors. Vol. 43 (7). P. 897-903 (2009).

[6] A.G. Alexenko, I.I. Shagurin. Microcircuitry (Radio and communication, Moscow, 1990).

[7] N.A. Avaev, Yu.E. Naumov, V.T. Frolkin. Basis of microelectronics (Radio and communication, Moscow, 1991).

[8] V.I. Lachin, N.S. Savelov. Electronics (Phoenix, Rostov-na-Donu, 2001).

[9] R. Pal, R. Pandey, N. Pandey, R.Ch. Tiwari. Single CDBA Based Voltage Mode Bistable Multivibrator and Its Applications. Circuits and Systems. Vol. 6 (11). P. 237-251 (2015).

[10] Z.Yu. Gotra. Technology of microelectronic devices (Radio and communication, Moscow, 1991).

[11] V.L. Vinetskiy, G.A. Kholodar', Radiative physics of semiconductors. ("Naukova Dumka", Kiev, 1979, in Russian).

[12] P.M. Fahey, P.B. Griffin, J.D. Plummer. Point defects and dopant diffusion in silicon. Rev. Mod. Phys. Vol. 61 (2). P. 289-388 (1989).

[13] M.L. Krasnov, A.I. Kiselev, G.I. Makarenko. Integral equations ("Science", Moscow, 1976).

[14] E.L. Pankratov. Dopant diffusion dynamics and optimal diffusion time as influenced by diffusioncoefficient nonuniformity Russian Microelectronics. 2007. V.36 (1). P. 33-39.

[15] E.L. Pankratov. Redistribution of dopant during annealing of radiative defects in a multilayer structure by laser scans for production an implanted-junction rectifiers. Int. J. Nanoscience. Vol. 7 (4-5). P. 187-197 (2008).

[16] E.L. Pankratov. Decreasing of depth of implanted-junction rectifier in semiconductor heterostructure by optimized laser annealing. J. Comp. Theor. Nanoscience. Vol. 7 (1). P. 289-295 (2010).

[17] E.L. Pankratov, E.A. Bulaeva. Optimization of manufacturing of emitter-coupled logic to decrease surface of chip. International Journal of Modern Physics B. Vol. 29 (5). P. 1550023-1-1550023-12 (2015).

[18] E.L. Pankratov, E.A. Bulaeva. An approach to manufacture of bipolar transistors in thin film structures. On the method of optimization. Int. J. Micro-Nano Scale Transp. Vol. 4 (1). P. 17-31 (2014). 
International Journal of Computational Science, Information Technology and Control Engineering

(IJCSITCE) Vol.3, No.3, July 2016

[19] E.L. Pankratov, E.A. Bulaeva. Increasing of sharpness of diffusion-junction heterorectifier by using radiation processing. Int. J. Nanoscience. Vol. 11 (5). P. 1250028-1250035 (2012).

[20] E.L. Pankratov, E.A. Bulaeva. Decreasing of mechanical stress in a semiconductor heterostructure by radiation processing. J. Comp. Theor. Nanoscience. Vol. 11 (1). P. 91-101 (2014).

\section{AUTHORS:}

Pankratov Evgeny Leonidovich was born at 1977. From 1985 to 1995 he was educated in a secondary school in Nizhny Novgorod. From 1995 to 2004 he was educated in Nizhny Novgorod State University: from 1995 to 1999 it was bachelor course in Radiophysics, from 1999 to 2001 it was master course in Radiophysics with specialization in Statistical Radiophysics, from 2001 to 2004 it was PhD course in Radiophysics. From 2004 to 2008 E.L. Pankratov was a leading technologist in Institute for Physics of Microstructures. From 2008 to 2012 E.L. Pankratov was a senior lecture/Associate Professor of Nizhny Novgorod State University of Architecture and Civil Engineering. 2012-2015 Full Doctor course in Radiophysical Department of Nizhny Novgorod State University. Since 2015 E.L. Pankratov is an Associate Professor of Nizhny Novgorod State University. He has 155 published papers in area of his researches.

Bulaeva Elena Alexeevna was born at 1991. From 1997 to 2007 she was educated in secondary school of village Kochunovo of Nizhny Novgorod region. From 2007 to 2009 she was educated in boarding school "Center for gifted children". From 2009 she is a student of Nizhny Novgorod State University of Architecture and Civil Engineering (spatiality "Assessment and management of real estate"). At the same time she is a student of courses "Translator in the field of professional communication" and "Design (interior art)" in the University. Since 2014 E.A. Bulaeva is in a PhD program in Radiophysical Department of Nizhny Novgorod State University. She has 103 published papers in area of her researches. 